\title{
Synthesis in Escherichia coli Cells and Characterization of the Active Exoribonuclease of Severe Acute Respiratory Syndrome Coronavirus ${ }^{1}$
}

\author{
P. Chen ${ }^{a, b}$, T. Hu ${ }^{a}$, M. Jiang ${ }^{a}$, and D. Guo ${ }^{a}$ \\ ${ }^{a}$ State Key Laboratory of Virology and Modern Virology Research Centre, College of Life Sciences, \\ Wuhan University, Wuhan 430072, PR. China \\ ${ }^{b}$ Department of Pathophysiology, Basic Medical College of Zhengzhou University, Zhengzhou 450001, PR. China \\ e-mail:dguo@shu.edu.cn \\ Received March 31, 2008 \\ Accepted for publication April 30, 2008
}

\begin{abstract}
The nsp14 protein, an exoribonuclease of the DEDD superfamily encoded by severe acute respiratory syndrome coronavirus (SARS-CoV), was expressed in fusion with different affinity tags. The recombinant nsp14 proteins with either GST fusion or 6-histidine tag were shown to possess ribonuclease activity but nsp14 with a short MGHHHHHHGS tag sequence at the N-terminus increased the solubility of nsp14 protein and facilitated the protein purification. Mutations of the conserved residues of nsp14 resulted in significant attenuation but not abolishment of the ribonuclease activity. Combination of fluorescence and circular dichroism spectroscopy analyses showed that the conformational stability of nsp14 protein varied with many external factors such as $\mathrm{pH}$, temperature and presence of denaturing chemicals. These results provide new information on the structural features and would be helpful for further characterization of this functionally important protein.
\end{abstract}

DOI: $10.1134 / \mathrm{S} 0026893309030091$

Key words: SARS coronavirus, exoribonuclease, affinity tag, mutagenesis, conformational stability

\section{INTRODUCTION}

Severe acute respiratory syndrome coronavirus (SARS-CoV) is the causative agent for the epidemic in year 2002 [1-3]. It is a positive-strand RNA virus whose gene expression and genome replication require polyprotein synthesis and subgenomic production [4-8]. Coronavirus replication requires several viral encoded proteins, including RNA-dependent RNA polymerase, RNA helicase, endoribonuclease, as well as a unique exoribonuclease [7, 9-10], The possible role of this exoribonuclease of SARS-CoV, nsp14, in the virus replication is reported [5, 9]. Although it has been proposed that nspl4 may play a role in the proof-reading, RNA repair and/or recombination for maintaining the integrity of the unusually large RNA genome of coronaviruses [7], the actual biological function of nsp14 involved in viral RNA replication is still unknown. To gain more insight into the biological functions and molecular mechanisms of nsp14, further characterization of its biochemical and conformational properties is needed.

Production of soluble recombinant proteins is vital for structure-function analysis. It is important to select

\footnotetext{
${ }^{1}$ The article is published in the original.
}

a proper vector and affinity tags for protein expression and purification [11]. Some tags such as maltose-binding protein (MBP) or glutathione S-transferase (GST) are used for both affinity purification and solubility. As mentioned previously MBP and GST have long been used to increase the solubility of complex proteins in Escherichia coli [11-13]. The hexahistidine tag (His6-tag) was widely used as affinity tag, its small size and the robust nature of the resin purification contributed to its popularity [11, 14].

In this study, the recombinant nsp14 of SARS-CoV was expressed in E. coli in three different forms and all of them possessed an active exoribonuclease activity, however, the nsp14 with a short modified MGHHHHHHGS tag exhibited higher solubility and facilitated the purification. Mutations to the conserved residues of nsp14 significantly attenuated the enzymatic activity. The conformational stability of nsp14 protein was analyzed by fluorescence and circular dichroism (CD) spectroscopy after exposure to various stressed conditions including different concentrations of denaturant, different $\mathrm{pH}$ and elevated temperature, and these biophysical analyses provided new information on the structural features of the SARS-CoV exoribonuclease. 
Oligonucleotides used for PCR amplification

\begin{tabular}{c|l}
\hline Primer usage & \multicolumn{1}{c}{ Primer sequence* } \\
\hline HT-nsp14 & F: 5'-agcccatgggccatcatcatcatcatcacggatccgcagaaaatgtaactgga-3' \\
HTL-nsp14\& & F.: 5'-ctgcagtcgacttactgtaacctggtaaatgt-3' \\
GST-nsp14 & R.: 5'-ctgcagtcgacttactgtaacctggtaaatgt-3' \\
D5992A\& & FM: 5'-tggattggctttgctgtagcgggctgtcatgca-3' \\
E5994A & RM: 5'-tgcatgacagcccgctacagcaaagccaatcca-3' \\
D6145A & FM: 5'- tttatgattgctgttcagcag-3' \\
H6170A & RM: 5'- ctgctgaacagcaatcataaa-3' \\
& FM: 5'- tggaaatgcacttgtggctagtt-3' \\
& RM: 5'- aactagccacaagtgcatttcca-3' \\
\hline
\end{tabular}

Note: $* \mathrm{~F}$ and $\mathrm{R}$ are, respectively, the forward and reverse primers for cloning into expression vector; FM and RM are, respectively, the forward and reverse primers used for site-directed mutagenesis.

\section{EXPERIMENTAL}

Construction of fusion nsp14 protein. The SARS-CoV cDNA was synthesized by reverse transcription using total RNAs extracted from Vero E6 cells infected by the virus isolate WHU (GenBank accession number AY394850), using poly d(T) primer. The $n s p 14$ gene was PCR amplified from the SARS-CoV cDNA using oligonucleotides shown in Table 1 and ligated into pGEX-4T-1 or pET-28a vector after digestion with BamHI and SalI. The resultant plasmid, pGST-nsp14, pHTL-nsp14, encoded SARSCoV nsp14 with a GST or His6 tag with the linker sequence from the vector in the $\mathrm{N}$-terminus. To remove the extra vector sequence, the PCR product was digested with $\mathrm{Ncol}$ and Sail and inserted into the NcoI and SalI sites of the vector pET-28a. The resulting plasmid, pHT-nsp14, encoded nsp14 with a MGHHHHHHGS sequence at the $\mathrm{N}$-terminus without other linker sequence.

Protein expression and purification. Plasmid pHT-nsp14, pHTL-nsp14 and pGST-nsp14 were transformed into E. coli BL21 (DE3) cells. Cultures were grown at $37^{\circ} \mathrm{C}$ until the $\mathrm{A}_{600}$ reached 0.8 and then induced with $0.5 \mathrm{mM}$ isopropyl-1-thio-D-galactopyranoside at $20^{\circ} \mathrm{C}$ for $16 \mathrm{~h}$. The GST fusion protein from pGST-nsp14 was purified using Glutathione Sepharose 4B resin (Amersham Biosciences) and His6 fusions from pHT-nsp14 and pHTL-nsp14 were purified by Ni-NTA agarose beads (Qiagen). Further purification was done by gel filtration chromatography over Superdex-75 (Amersham Biosciences). For gel filtration experiments, the column was equilibrated with PBS and eluted with the same buffer. The purified protein was confirmed by sodium dodecyl sulfate-polyacrylamide gel electrophoresis (SDSPAGE) and mass spectrometry. The amount of protein was measured using BioRad protein assay kit.

Preparation of RNA substrates. A chemically synthesized, hairpin RNA-encoding DNA sequence
(GGATCCCGCATTCTATCCTCTAGAGGATGTTCAAGAGACATCCTCTAGAGGATA GAATGTTTTTTGGAAAAGCTT) was ligated into the BamHIHindIII site of pCR3.1 vector. The DNA construct was digested with EcoRI and transcribed by T7 RNA polymerase. The RNA was purified from denaturing $7 \mathrm{M}$ urea-8\% polyacrylamide gels (19:1 of acrylamid to bisacrylamide) and labeled at the 5'-end with T4 polynucleotide kinase and $\left[\gamma^{-32} \mathrm{P}\right] \mathrm{ATP}$. After the labeling, the RNA was purified using G-25 column.

Site-directed mutagenesis. Site-directed mutagenesis was done by splicing via overlap extension (SOE) PCR [15]. Nucleotide sequences of the primers used for site-directed mutagenesis are given in Table 1. The sequences of all the constructs were confirmed by DNA sequence analysis. The correct plasmids encoding mutant forms of nsp14 were then transformed into E. coli BL21 (DE3) cells and the recombinant proteins were expressed and affinity purified as described above. The purity of the mutant proteins was analyzed by SDS-PAGE.

Exonuclease assays. The standard RNA nuclease assay was carried out as previously described [16-19]. Standard reaction volume including $1 \mathrm{kBq}$ of radiolabeled RNA substrate and $2.3 \mu \mathrm{M}$ of nsp14 in a buffer consisting of $50 \mathrm{mM}$ Tris- $\mathrm{HCl}$ (pH 8.0), $50 \mathrm{mM} \mathrm{KAc}$, $2 \mathrm{mM}$ dithiothreitol, $10 \%$ glycerol, $0.1 \mathrm{mg} / \mathrm{ml} \mathrm{BSA}$, $5 \mathrm{mM} \mathrm{MgAc} 2,25 \mathrm{mM} \mathrm{NaH} \mathrm{PO}_{4}$. The reaction mixture was incubated at $37^{\circ} \mathrm{C}$ for 2 min unless stated otherwise. Reactions were terminated by the addition of the equal volumes of gel-loading buffer (containing $96 \%$ formamide and $1 \mathrm{mM}$ EDTA). The mixture was heated to $85^{\circ} \mathrm{C}$ for $2 \mathrm{~min}$ and put to ice bath immediately. Products were analyzed by denaturing gels composed of $7 \mathrm{M}$ urea and $8 \%$ polyacrylamide. Gels were dried and exposed to a Phosphorlmager screen (Amersham Biosciences).

Circular Dichroism spectroscopy. The circular dichroism (CD) spectra were recorded using a Jasco 
(a)

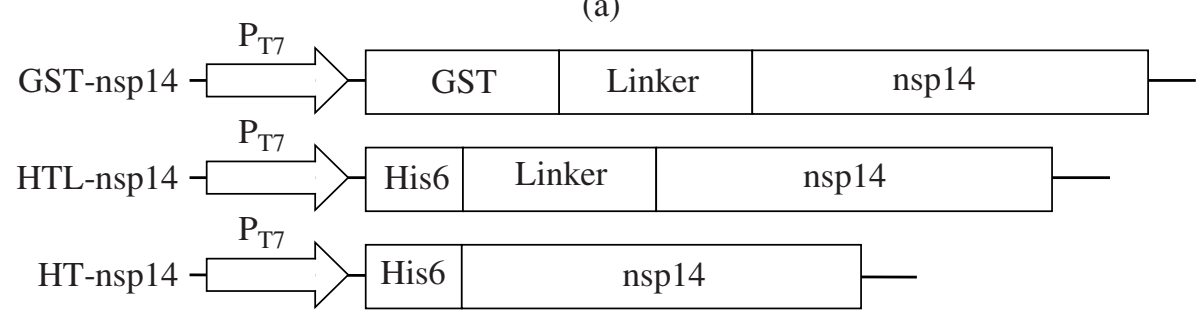

(b)

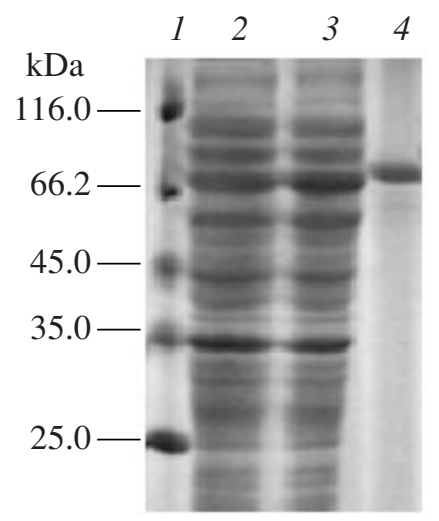

(c)

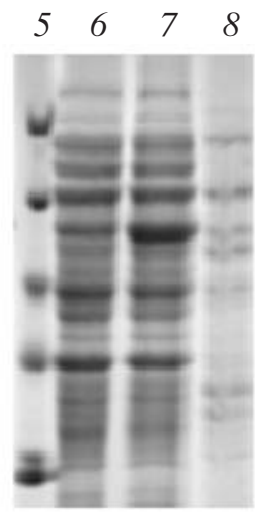

(d)

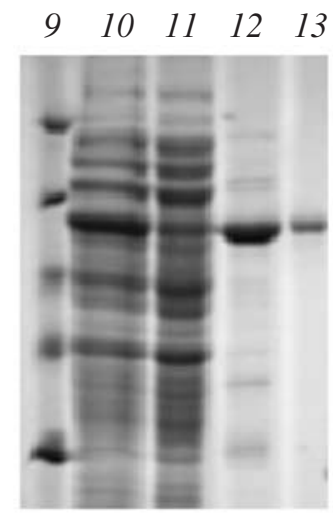

Fig. 1. Expression of recombinant SARS-CoV nsp14. (a) Schematic representation of the different strategies of expressing nsp14 protein. (b) SDS-PAGE of the GST-nsp14 protein purified from E. coli cells. Lane 1: molecular weight makers, Lane 2: GST-nsp14 supernatant after sonication, Lane 3: GST-nsp14 pellet after sonication, Lane 4: protein eluted from GST affinity column. (c) SDSPAGE of the HTL-nsp14 protein purified from E. coli cells. Lane 5: molecular weight makers, Lane 6: HTL-nspl4 supernatant after sonication, Lane 7: HTL-nsp14 pellet after sonication, Lane 8: HTL-nsp14 eluted from NTA-Ni ${ }^{2+}$ affinity column. (d) SDS-PAGE of the HT-nsp14 protein purified from E. coli cells. Lane 9: molecular weight makers, Lane 10: HT-nsp14 supernatant after sonication, Lane 11: HT-nsp14 pellet after sonication, Lane 12: HT-nsp14 eluted from NTA-Ni ${ }^{2+}$ affinity column, Lane 13: the HT-nsp14 purified by gel filtration chromatography on Superdex-75 column.

$\mathrm{J}-810$ spectropolarimeter in $40 \mathrm{mM}$ Tris- $\mathrm{HCl}$ buffer $(\mathrm{pH} 7.2)$ at $20^{\circ} \mathrm{C}$. A cell with a path length of $0.1 \mathrm{~mm}$ was used for far-UV CD spectrum. Each spectrum was the average of four scans corrected by subtracting a spectrum of the buffer solution in the absence of protein recorded under identical condition. Each scan in the range of 200-250 $\mathrm{nm}$ was obtained by taking data points every $0.5 \mathrm{~nm}$ with integration time of $1 \mathrm{~s}$ and a $2 \mathrm{~nm}$ bandwidth.

Fluorescence measurements. All fluorescence measurements were performed in a RF-5301PC speetrofluorophotometer. Slit widths with a nominal band pass of $3 \mathrm{~nm}$ were used for both excitation and emission beams. Intrinsic tluorescencc emission spectra were recorded from 300 to $500 \mathrm{~nm}$ after exciting at 275,280 and $295 \mathrm{~nm}$. The emission intensity value at $\sim 343 \mathrm{~nm}$ was evaluated in each case from the scans.

\section{RESULTS AND DISCUSSION Expression of SARS-CoV nsp14 Protein}

To facilitate biochemical and biophysical analyses of SARS-CoV nsp14 protein, we first optimized the expression and purification conditions for this protein.
As shown in Fig. 1, SARS-CoV nsp14 was expressed in $E$. coli with a $6 \times$ His or GST tag fused at the N-terminus in different forms. The GST-nsp14 contained a large part of GST fusion while HTL-nsp14 carried a $6 \times$ His tag with a long vector sequence. As shown in Fig. 1b, GST-nsp14 protein showed 30\% solubility when expressed at $20^{\circ} \mathrm{C}$ (lanes $2-3$ in Fig. 1b) and it could be purified by GST-affinity chromatography (lane 4 in Fig. 1b). To obtain an intact protein without extra sequence, we used thrombin protease to remove the GST tag after purification but the protein became easily to aggregate in solution after losing the GST tag (data not shown). The HTL-nsp14 protein with His6 tag and a long linker sequence from the vector at the $\mathrm{N}$-terminus was produced mostly in the form of inclusion body and was poorly purified by Ni-NTA affinity column (lanes 6-8 in Fig. 1c).

As both GST-nsp14 and HTL-nsp14 had problems with either stability or solubility, we expressed nsp14 with a short tag (MGHHHHHHGS) in the N-terminus by removing all other vector sequence contained in HTL-nsp14, and resulted in the recombinant protein HT-nsp14. A significant increase in yield and solubility (about 50\%) was observed with HT-nsp14 
(a)

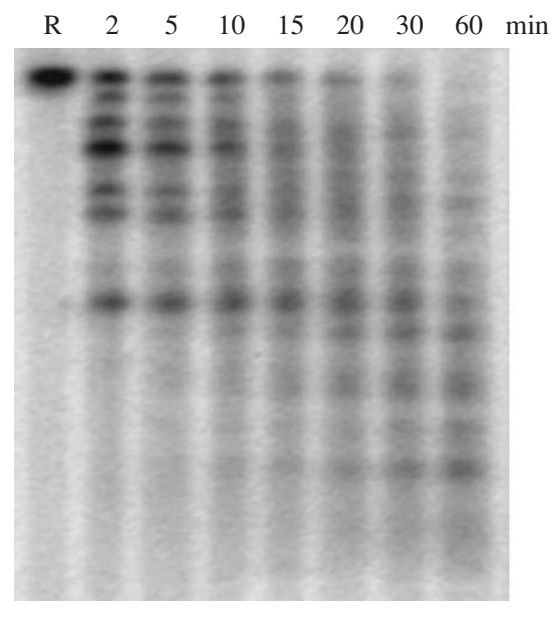

(c)

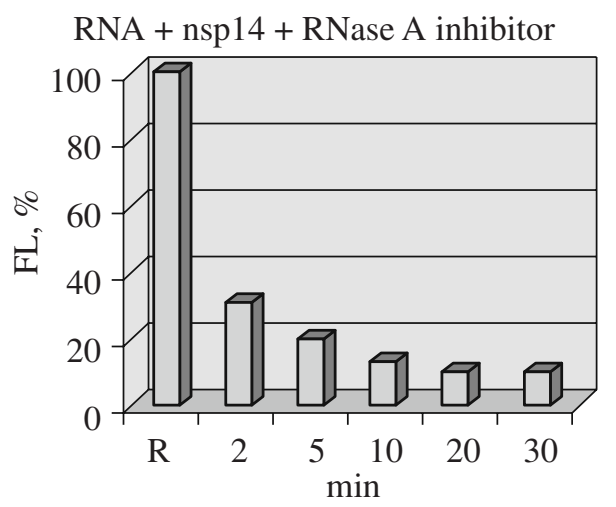

(b)

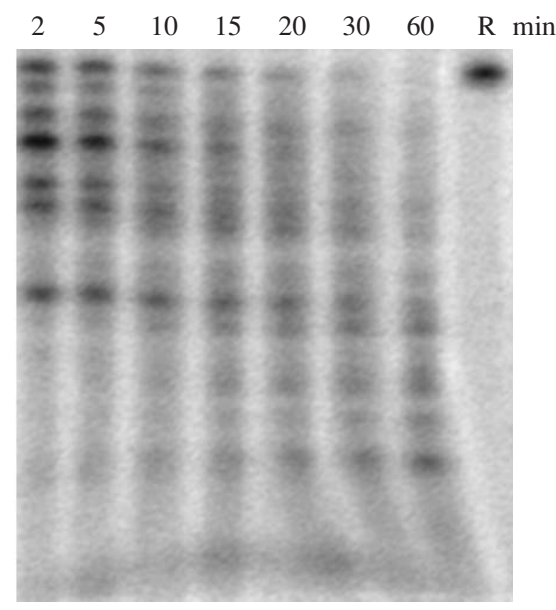

(d)

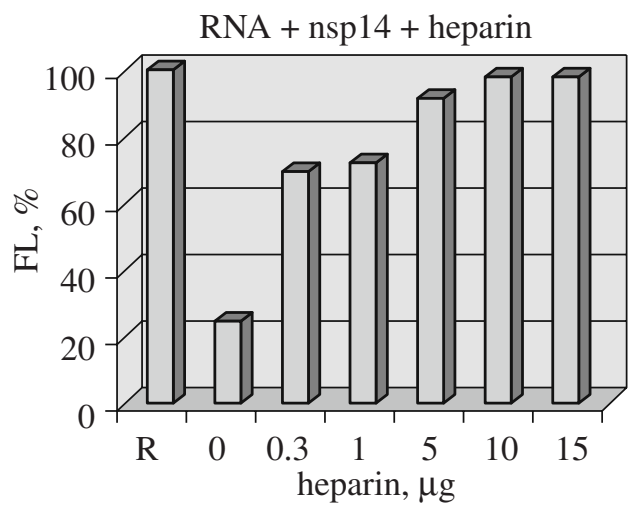

Fig. 2. The exoribonuclease activity associated with the purified HT-nsp14 (a) and GST-nsp14 (b). The RNA substrate was 5'-[ $\left.{ }^{32} \mathrm{P}\right]-$ radiolabeled. The incubation time is shown above the lanes. The control RNA incubated without nsp14 is marked R. (c) Effect of RNaseA inhibitor on cleavage of 5'-radiolabeled RNA by nsp14. The exoribonuclease reaction was performed in the same buffer conditions as described in Fig. 2a, except that $400 \mathrm{U}$ of RNaseA inhibitor was present. The reaction was carried out at the time points indicated in the horizontal axis. (d) Effect of heparin on cleavage of 5'-labeled hairpin RNA by nsp14. RNA digestion was performed in the absence or presence of various concentrations of heparin. Uncleaved full-length substrate RNA was quantified and shown as a percentage of input full-length RNA ([FL (\%)]).

(lanes $10-11$ in Fig. 1d). After initial purification on a Ni-NTA affinity column, the purity of HT-nsp14 reached $90 \%$, and with an additional step of gel filtration chromatography on Superdex-75 column (Amersham, Inc), the protein was purified to near homogeneity (lanes $12-13$ in Fig. 1d). This tag is very small, which does not need removal of the tag during the further structural and functional studies, in fact for structural studies, more than $60 \%$ of the proteins produced include a polyhistidine tag, and many examples of proteins crystallized with His-tag left intact are reported [13]. At the same time, Gly-Ser-coding sequences can be made to contain Bam HI or BglII sites and this can facilitate the cloning work. During the following experiments, the analyses of the conformational stability of nsp14 were performed with HTnsp14.

\section{The Ribonuclease Activity of SARS-CoV Protein nspl4}

To determine whether the different forms of the recombinant nsp14 protein had the ribonuclease activity, the protein was incubated with the 5'-radiolabeled RNA molecules that was obtained by in vitro transcriptional system. As shown in Fig. $2 a$ and $2 b$, the RNA substrate was digested to many small fragments with similar patterns by either GST-nsp14 or HTnsp14. The digestion of the RNA became more extensive and the product fragments became smaller as the incubation time was longer. These results suggested that both GST-fused and 6His-tagged nsp14 proteins possessed a ribonuclease activity.

To rule out the possibility that the ribonuclease activity was from the contaminated RNase A that was 
(a)

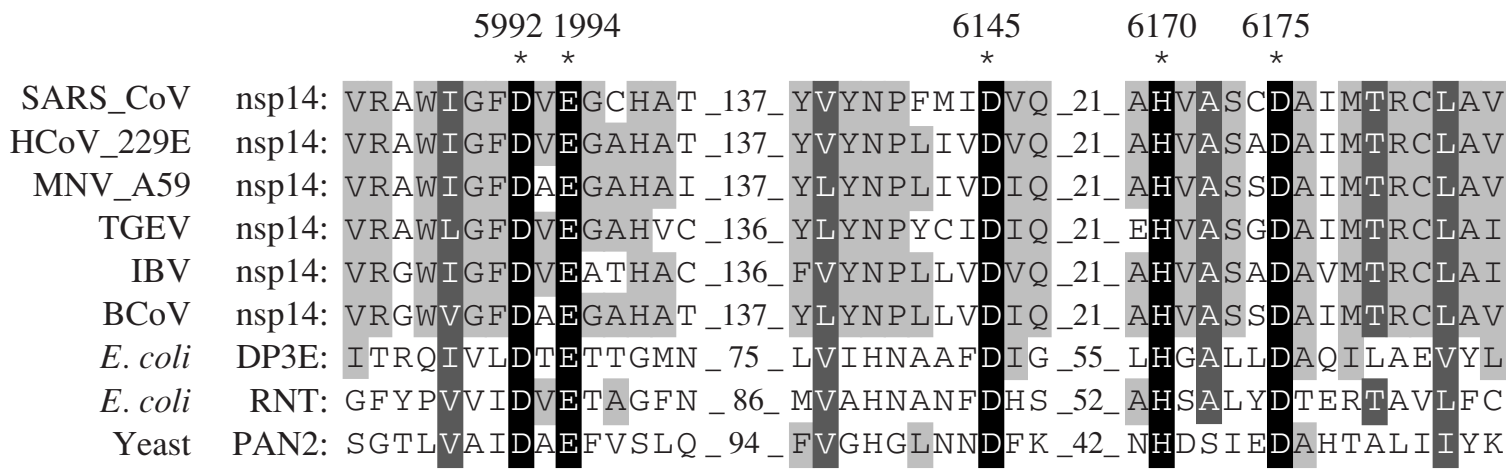

(b)

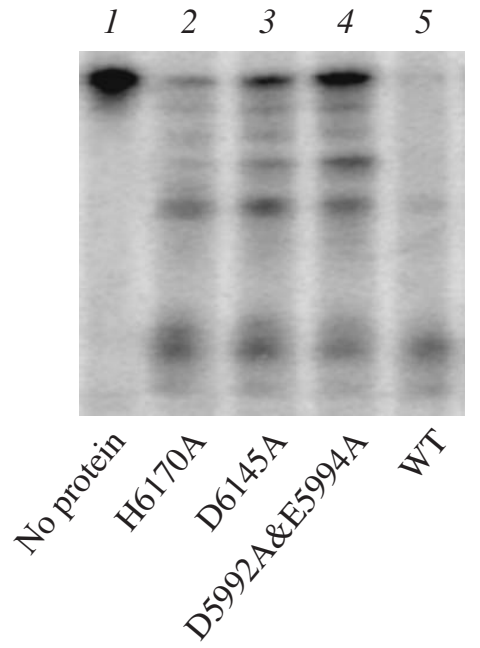

(c)

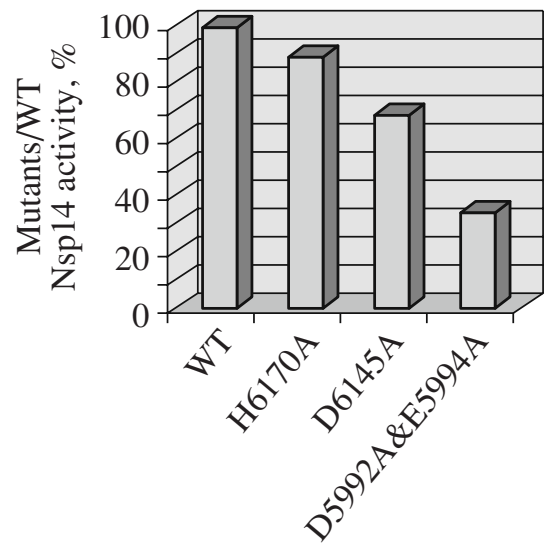

Fig. 3. Mutational analysis of nsp14 exoribonuclease. (a) Sequence alignment of nsp14 with its homolog, showing the regions containing the highly conserved residues that are the putative active center amino acids. The five highly conserved residues predicted to be important for activity are indicated by *. Four of these five residues were mutated and their activity was tested as shown in panel b. (b) Exoribonuclease activity of the nsp14 mutants. Lane 1 contains only RNA substrate. The mutants and wildtype (WT) nsp14 are indicated in Lanes $2-5$. The exoribonuclease activity assay was performed using the condition as described in Fig. 2 a. The incubation time for the reaction was $60 \mathrm{~min}$ at $37^{\circ} \mathrm{C}$. (c) A chart quantify the activity of the mutants as \% of wildtype (WT), as shown in panel (b).

co-purified, we added the RNase A inhibitor to the same reaction mixture. The result showed no inhibition of the RNA digestion by RNase A inhibitor (Fig. 2c), suggesting that no RNase A activity was involved in digesting the RNA substrate. On the other hand, the heparin is a competitive inhibitor of nucleic acid binding and nucleic acid binding is a prerequisite for nuclease activity $[20,21]$. Therefore, we tested the influence of heparin on the nsp14 enzymatic activity and the results showed that heparin could inhibit the exonuclease activity in concentration-dependent manner (Fig. 2d).

\section{Mutational Analysis of nsp14}

SARS-CoV nsp14 and other members of the DEDD superfamily proteins have a characteristic four acidic amino acids that are absolutely conserved (D5992, E5994, D6145, D6175), plus a highly conserved residue of H6170 (Fig. 3a) [22]. We carried out the point mutations of the four residues, D5992/E5994, D6145, and H6170, all to Ala, to examine the functional role of these residues. The results showed that each mutation had significant impact on the enzymatic activity and all mutants displayed significantly lower ribonuclease activity than the wildtype protein (Fig. 3b and 3c), demonstrating the critical rote of these conserved residues. Nonetheless, all these mutants still retained low level of exoribonuclease activity, and a complete abolishment of the activity may require a combination of two or more of these mutations. 
(a)

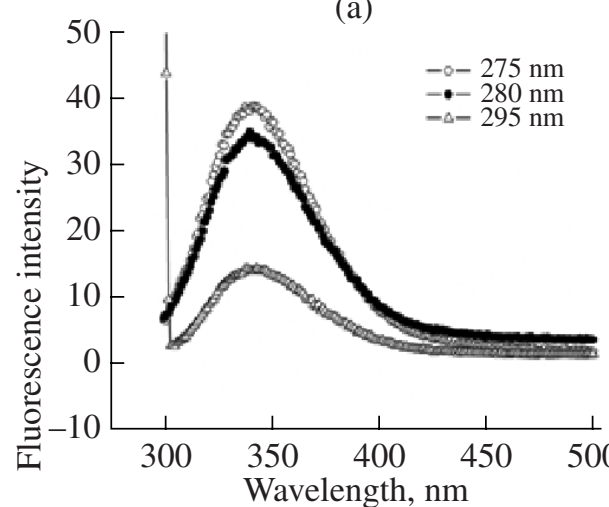

(c)

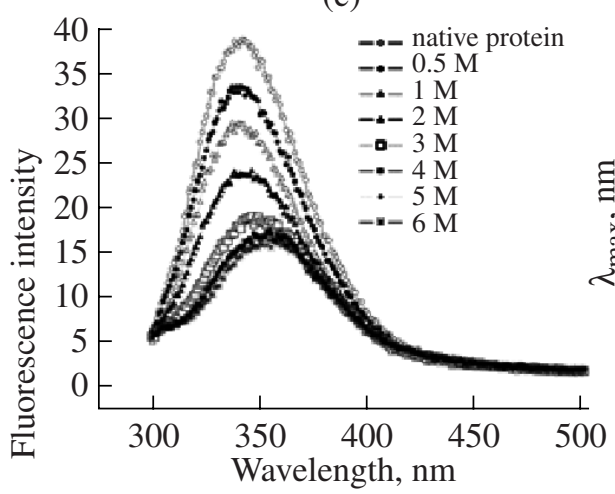

(b)

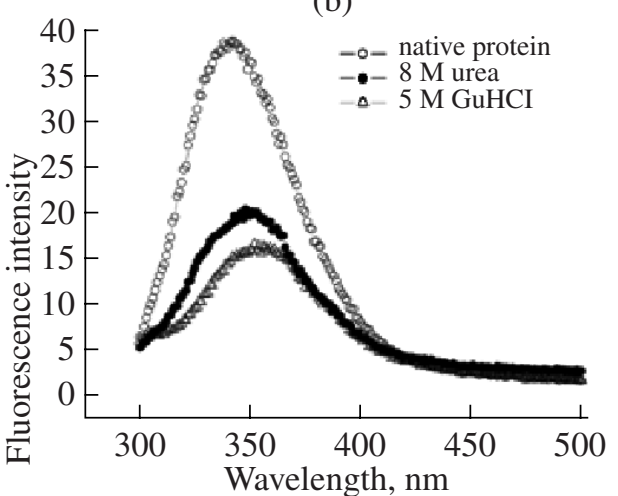

(d)

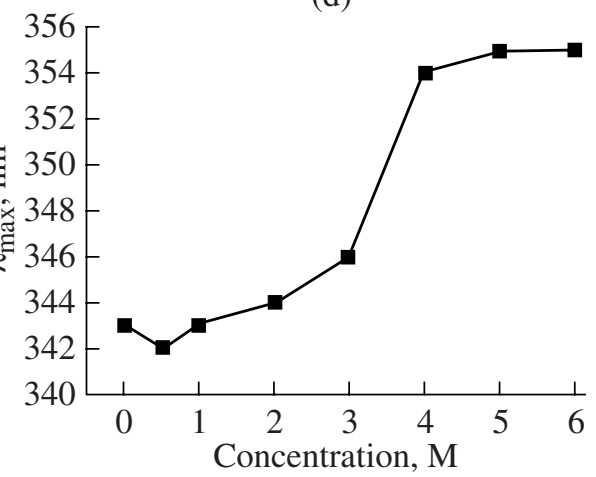

Fig. 4. (a) Fluorescence emission spectral of nsp14 under different excitation. (b) Fluorescence emission spectral of nsp14 with different denaturants. (c) Fluorescence spectrum of nsp14 under different concentrations of GuHCl. (d) Wavelength of maximum emission spectrum of nsp14 under different concentration of $\mathrm{GuHCl}$.

\section{Fluorescence Spectrum Characterization of nsp14}

Fluorescence emission spectra obtained for HTnsp14 (Fig. 4a) are typical of those generally observed for Trp-containing proteins [23], despite of relatively high content of Tyr residues within the studied protein molecules. Nsp14 has similar fluorescence emission spectrum using different excitation spectrum and has maximum emission spectrum at about $343 \mathrm{~nm}$. The fluorescence intensity is lower at $296 \mathrm{~nm}$ than that at 275 and $280 \mathrm{~nm}$ (Fig. 4a). The maximum emission spectrum of the enzyme $(343 \mathrm{~nm})$ is blue shifted relative to that of free L-tryptophan, which is observed to be at $354 \mathrm{~nm}$ under the same conditions, indicating that the tryptophan residues in nspl4 are not completely exposed to the solvent. This shielding of the tryptophan residues from the solvent phase is the result of the folding and three-dimensional structure of the protein.

\section{Effect of Denaturant on the Stability of nsp14}

Tryptophan residues in native proteins are not found in identical locations, nor are they equally influenced by the environment. The microenvironment of every residue is characterized by a particular set of physico-chemical conditions (polarizability, microviscosity, availability of charged groups, possible specific interactions, etc.) that influence chromophore fluorescence. As a consequence, the protein fluorescence is conditioned by the sum of fluorescent contributions of individual tryptophan residues, which vary over a rather wide range [24].

As it is observed in Fig. 4a, the maximum wavelength $(\lambda \max )$ of nsp14 tryptophan fluorescence was $343 \mathrm{~nm}$. When the protein is in the presence of $8 \mathrm{M}$ urea and $6 \mathrm{M} \mathrm{GuHCl}$-containing aqueous solution, $\lambda \max$ had a large red-shifting to $351 \mathrm{~nm}$ and $354 \mathrm{~nm}$ (Fig. 4b). This red shifting may be explained in terms of increased exposure of Trp residues to solvent, resulting from the process of protein denaturation [23, 25]. The red shift in emission maxima was also accompanied by an increase in the half-maximal spectral width of the fluorescence spectra (Fig. 4b). This increase was largely due to the emergence and contribution of tyrosine residues to the emission spectrum upon denaturation and the formation of more conformationally altered species during the denaturation process [26].

The fluorescence spectra of native nsp14 showed maximum emission spectrum at $343 \mathrm{~nm}$. Incubation of nsp14 at increasing concentrations of $\mathrm{GuHCl}$ 


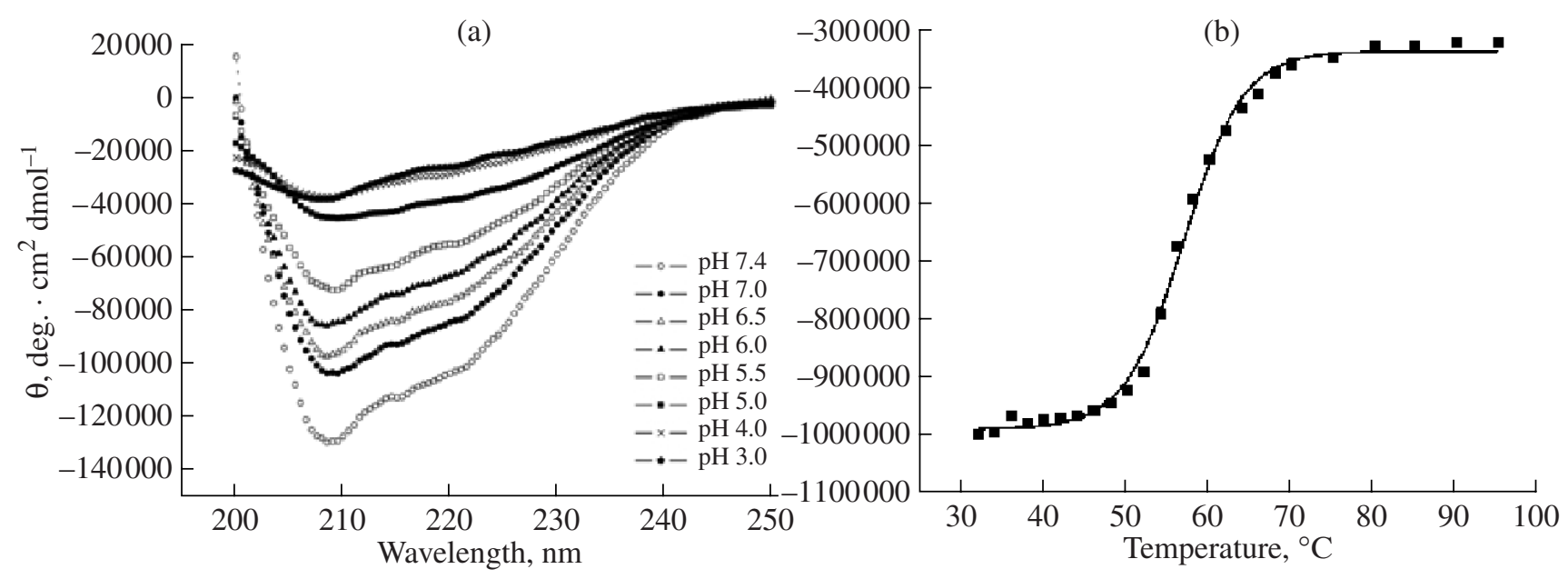

Fig. 5. Circular dichroism spectrum analysis of nsp14. (a) Effect of pH on the secondary structure of nsp14. (b) The thermal unfolding followed at $222 \mathrm{~nm}$ with a temperature range from 30 to $95^{\circ} \mathrm{C}$.

resulted in a progressive change of intrinsic fluorescence emission to a longer wavelength, $354 \mathrm{~nm}$ (Fig. 4c and 4d). This is due to the fact that the Trp residue, which is partly buried in the native form, is exposed on the surface in the denatured protein. At $\mathrm{GuHCl}$ concentrations higher than $4 \mathrm{M}$ the fluorescence intensity measured at $343 \mathrm{~nm}$ drops to about $40 \%$ of its value observed in solutions with no $\mathrm{GuHCl}$ present (Fig. 4c). This decrease of fluorescence intensity obviously results from the denaturation of protein and may be explained in terms of increasing polarity of the Trp environment as it becomes fully exposed to the solvent.

\section{Effect of $\mathrm{pH}$ and Temperature on the Structure of nsp14}

To examine the $\mathrm{pH}$ and thermal stability of nsp14, we recorded circular dichroism spectra under a changing condition (Fig. 5). The spectrum of nsp14 at room temperature $\left(22^{\circ} \mathrm{C}\right)$ displays a $\alpha$-helical band with double negative ellipticity at $222 \mathrm{~nm}$ and $208 \mathrm{~nm}$. At the same time, slightly different from the classical helical spectrum, the spectrum of nsp14 showed that the mean residue ellipticity at $208 \mathrm{~nm}$ was of substantially higher magnitude than that at $222 \mathrm{~nm}$, which indicated that nsp14 is a protein mainly consists of $\alpha$ helices and $\beta$-sheets; belong to the protein class of $\alpha+$ $\beta$ (Fig. 5a, the bottom line) [27].

The effect of changing $\mathrm{pH}$ condition on the secondary structure of nsp14 is shown in Fig. 5a. By shifting the $\mathrm{pH}$ in the range $\mathrm{pH} 3$ to 7.4 , the $\alpha$-helix is reduced with lower $\mathrm{pH}$. The far-UV CD spectra revealed a clearly difference below $\mathrm{pH} 5.5$, suggesting a structural transition from a $\beta$-helix structure to a slightly more $\beta$-sheet structure. It has been observed that ahelices may undergo structural transition in acidic solution and at elevated temperatures [28].
The thermal stability of nsp14 was assessed by monitoring the changes in the $\alpha$-helix content of the protein at $222 \mathrm{~nm}$ during the temperature range from 30 to $95^{\circ} \mathrm{C}$. In the interval from 30 to $50^{\circ} \mathrm{C}$, the $\mathrm{CD}$ spectrum of nsp14 was steady. Under further heating from 50 to $75^{\circ} \mathrm{C}$, the $\mathrm{CD}$ spectrum underwent a change to another pattern with approximately three-fold lower the mean residue ellipticity at $222 \mathrm{~nm}$. These data indicated that the helical structure of nsp14 was disrupted by the thermal treatment. The result shown in Fig. 5b indicated that the Tm value of nsp14 is approximately $58^{\circ} \mathrm{C}$.

Protein folding is a central problem in biochemistry and has continued to receive considerable attention. Proper evaluation of protein's stability and factors that contribute to the conformational preference for native form relative to unfolded form(s) are critical for understanding protein folding and function. The results presented here provide some straightforward information about the structural stability of nsp14, which may provide the basis for further studies about biological functions of SARS-CoV nsp14.

\section{ACKNOWLEDGMENTS}

We thank $\mathrm{Mu}$ Xiao, Zhiqiang Chen and Peng Zhang for their help and Dr Yi Liang for advice in some of the experiments.

This study was supported by China National Science Foundation (no. 30570394), National Basic Research Program of China (no.2006CB504300) and the MOE "111" project no.B06018.

\section{REFERENCES}

1. Drosten C., Gunther S., Preiser W., et al. 2003. Identification of a novel coronavirus in patients with severe 
acute respiratory syndrome. N. Engl.J. Med.348, 19671976.

2. Fouchier R.A., Kuiken T., Schutten M., van Amerongen G., van Doornum G.J., van den Hoogen B.G., Peiris M., Lim W., Stohr K., Osterhaus A.D. 2003. Aetiology: Koch's postulates fulfilled for SARS virus. Nature. 423, 240.

3. Ksiazek T.G., Erdman D., Goldsmith C.S., et al. 2003. A novel coronavirus associated with severe acute respiratory syndrome. N. Engl. J. Med. 348, 1953-1966.

4. Hussain S., Pan J., Chen Y., Yang Y., Xu J., Peng Y., Wu Y., Li Z., Zhu Y., Tien P., Guo D. 2005. Identification of novel subgenomic RNAs and noncanonical transcription initiation signals of severe acute respiratory syndrome coronavirus. J. Virol. 79, 5288-5295.

5. Chen P., Jiang M., Hu T., Liu Q., Chen S.X., Guo D. 2007. Biochemical characterization of exoribonuclease encoded by SARS coronavirus. J. Biochem. Mol. Biol. 40, 649-655.

6. Ruan Y.J., Wei C.L., Ee A.L., et al. 2003. Comparative full-length genome sequence analysis of 14 SARS coronavirus isolates and common mutations associated with putative origins of infection. Lancet. 361, 1779-1785.

7. Snijder E.J., Bredenbeek P.J., Dobbe J.C., Thiel V., Ziebuhr J., Poon L.L., Guan Y., Rozanov M., Spaan W.J., Gorbalenya A.E. 2003. Unique and conserved features of genome and proteome of SARS-coronavirus, an early split-off from the coronavirus group 2 lineage. J. Mol. Biol. 331, 991-1004.

8. Thiel V., Ivanov K.A., Putics A., et al. 2003. Mechanisms and enzymes involved in SARS coronavirus genome expression. J. Gen. Virol. 84, 2305-2315.

9. Minskaia E., Hertzig T., Gorbalenya A.E., Campanacci V., Cambillau C., Canard B., Ziebuhr J. 2006. Discovery of an RNA virus $3^{\prime} \rightarrow 5^{\prime}$ exoribonuclease that is critically involved in coronavirus RNA synthesis. Proc. Natl. Acad. Sci. USA. 103, 5108-5113.

10. Ziebuhr J. 2005. The coronavirus replicase. In: Coronavirus Replication and Reverse Genetics. Ed. Enjuanes L. Berlin: Springer, pp. 58-94.

11. Arnau J., Lauritzen C., Petersen G.E., Pedersen J. 2006. Current strategies for the use of affinity tags and tag removal for the purification of recombinant proteins. Protein Expr. Purif. 48, 1-13.

12. Smith D.B., Johnson K.S. 1988. Single-step purification of polypeptides expressed in Escherichia coli as fusions with glutathione S-transferase. Gene. 67, 31-40.

13. Derewenda Z.S. 2004. The use of recombinant methods and molecular engineering in protein crystallization. Methods. 34, 354-363.

14. Smith M.C., Furman T.C., Ingolia T.D., Pidgeon C. 1988. Chelating peptide-immobilized metal ion affinity chromatography: A new concept in affinity chromatography for recombinant proteins. J. Biol. Chem. 263, 72117215.

15. Horton R.M., Hunt H.D., Ho S.N., Pullen J.K., Pease L.R. 1989. Engineering hybrid genes without the use of restriction enzymes: Gene splicing by overlap extension. Gene. 77, $61-68$.

16. Astrom J., Astrom A., Virtanen A. 1992. Properties of a HeLa cell 3' exonuclease specific for degrading poly(A) tails of mammalian mRNA. J. Biol. Chem. 267, 1815418159 .

17. Caruccio N., Ross J. 1994. Purification of a human polyribosome-associated $3^{\prime}$ to $5^{\prime}$ exoribonuclease. $J$. Biol. Chem. 269, 31814-31821.

18. Chekanova J.A., Dutko J.A., Mian I.S., Belostotsky D.A. 2002. Arabidopsis thaliana exosome subunit AtRrp4p is a hydrolytic $3^{\prime} \rightarrow 5^{\prime}$ exonuclease containing $\mathrm{S} 1$ and $\mathrm{KH}$ RNA-binding domains. Nucleic Acids Res. 30, 695-700.

19. del Cardayre S.B., Raines R.T. 1994. Structural determinants of enzymatic processivity. Biochemistry. 33, 6031-6037.

20. Bhardwaj K., Guarino L., Kao C.C. 2004. The severe acute respiratory syndrome coronavirus Nsp 15 protein is an endoribonuclease that prefers manganese as a cofactor. J. Virol. 78, 12218-12224.

21. Reuven N.B., Weller S.K. 2005. Herpes simplex virus type 1 single-strand DNA binding protein ICP8 enhances the nuclease activity of the UL12 alkaline nuclease by increasing its processivity. J. Virol. 79, 9356-9358.

22. Zuo Y., Deutscher M.P. 2001. Exoribonuclease superfamilies: structural analysis and phylogenetic distribution. Nucleic Acids Res. 29, 1017-1026.

23. Lakowicz J.R. 1999. Principles of Fluorescence Spectroscopy. 2nd ed. N.Y.: Kluwer, pp. 445-486.

24. Burstein E.A., Vedenkina N.S., Ivkova M.N. 1973. Fluorescence and the location of tryptophan residues in protein molecules. Photochem. Photobiol. 18, 263-279.

25. Deshpande S.S., Damodaran S. 1991. Denaturation behavior of phaseolin in urea, guanidine hydrochloride, and sodium dodecyl sulfate solutions. J. Protein Chem. 10, 103-115.

26. King L. 1994. Effects of denaturant and pressure on the intrinsic fluorescence of titin. Arch. Biochem. Biophys. 311, 251-257.

27. Levitt M., Chothia C. 1976. Structural patterns in globular proteins. Nature. 261, 552-558.

28. Johnson W.C., Jr. 1988. Secondary structure of proteins through circular dichroism spectroscopy. Annu. Rev. Biophys. Biophys. Chem. 17, 145-166. 